\title{
Cognitive consonance: complex brain functions in the fruit fly and its relatives
}

\author{
Ralph J. Greenspan and Bruno van Swinderen
}

The Neurosciences Institute, 10640 John Jay Hopkins Drive, San Diego, CA 92121, USA

\begin{abstract}
The fruit fly, Drosophila melanogaster, has become a model for the study of a growing number of human characteristics because of the power of its genetics. Higher cognitive functions, however, might be assumed to be out of reach for the little fly. But the cumulative history of cognitive studies in insects and some of their arachnid relatives, as well as specific probing of the capabilities of fruit flies, suggests that even in this ethereal realm these creatures have much to contribute. What are the degrees of sophistication in cognitive behavior displayed by these organisms, how have they been demonstrated, and what is their potential for understanding how our own brains work?
\end{abstract}

Beginning with Aristotle, continuing through Descartes, and ultimately getting the imprimatur of hard science from Werner Reichardt, insects (and invertebrates generally) have been considered to be automata - robot-like creatures whose behavior appears mechanical, stereotypical and (in current parlance) rigidly programmed. This view carried with it the natural assumption that one need not waste any time trying to discern cognitive functions in these organisms. Through the long and arduous efforts of a handful of investigators, insects have gained ground in the struggle for cognitive legitimacy. The lion's share of this progress has come from studies of learning in relation to foraging and navigation in honeybees. More recently, studies of problem solving in the jumping spider Portia, an arachnid whose nervous system resembles that of insects, have further established the cognitive credentials of this corner of the invertebrate world. (The octopus, undisputed genius of the sub-kingdom, will be omitted from this discussion owing to its lack of anatomical similarity to insects and jumping spiders.) And, finally, the genetic potential of the fruit fly, Drosophila melanogaster, is now causing its capabilities to be probed far beyond what would seem reasonable for a creature with such apparently modest endowments, and it too is exceeding expectations for cognitive sophistication.

Learning tests have been the major vehicle for determining cognitive ability in these animals. This makes sense given that learning forms part of the definition of cognition. But these tests have also been the mainstay for studies of perception, which need not necessarily involve learning. Because animals cannot report their perceptions in any way other than behavior,

Corresponding author: Ralph J. Greenspan (greenspan@nsi.edu).

Available online 14 October 2004 the ability to be conditioned associatively with a given conditioned stimulus (CS) becomes the most straightforward measure of recognition (i.e. perception) for that stimulus.

\section{The honeybee}

The sophistication of honeybee cognition was first suggested by von Frisch's pioneering studies of honeybee foraging. He demonstrated not only that scouts have the ability to translate their experience of finding a nectar source into a sophisticated set of signals, the 'dance', but also that the observers of this dance have the ability to translate it into a sequence of navigational maneuvers [1]. These abilities are suggestive of the presence of explicit memory, a high-level cognitive function that involves memory of places, facts and events [2]. Since these studies, after a certain amount of controversy [3,4], the existence of a map-like, spatial memory in the honeybee has been formally demonstrated $[5,6]$.

The key to unlocking this behavior in bees was not over-training them to a single site. When over-trained to one site, they home reliably from that site and not very well from others. When lightly and variably trained to a variety of sites, they are far better at finding their way back to the hive from a range of locations, including some from which the hive is not visible [5]. This deleterious effect of overtraining has its neural counterpart in monkey studies showing a loss of normal organization in sensory cortex as a consequence of overtraining with monotonous stimuli $[7,8]$. Further confirmation of the ability of honeybees to choose a novel, shortest-path route comes from recent tests involving their release from unexpected sites and monitoring of their complete flight paths by using a harmonic radar system [6]. With the demonstration of map learning, the honeybee becomes a contender for possessing explicit memory. Implicit memory (motor memory or memory that deals with procedures) is unquestionably present, as evidenced by the extensive studies of associative conditioning, second-order conditioning, blocking, and contextual learning [9].

The standard laboratory tests for explicit memory in rodents are maze and place learning [2]. Place learning consists of associative conditioning using place cues as the CS. An analogous place-learning paradigm has been developed for the cockroach in which a plate is heated over its entire surface except for an unmarked cooled zone, and the plate is surrounded by place-specific visual patterns [10]. Place learning in these insects is not as robust as in rodents but the majority of animals do learn, and this 
learning is impaired in animals which had received lesions to portions of the mushroom bodies (the pedunculi at their junction with the $\beta$-lobes).

\section{The jumping spider}

Anticipatory maze learning has been demonstrated in salticid jumping spiders of the genus Portia. These animals are presented with a maze that can be viewed in its entirety from the vantage point of the spider. The maze consists of a set of wire walkways representing potential paths from the starting position to that of a food lure placed at the maze endpoint (Figure 1). One route reaches the food but the other does not. After scanning of the entire maze, visually following the tracks back from the food source, the spider chooses an entry point to the maze, choosing correctly in $75 \%$ of first time trials $[11,12]$. This remarkable display of problem solving is carried out by a creature with a brain several hundred microns in diameter. Salticid spiders share with insects a rough similarity in body plan and size, and they have a complex brain with structures that somewhat resemble those of

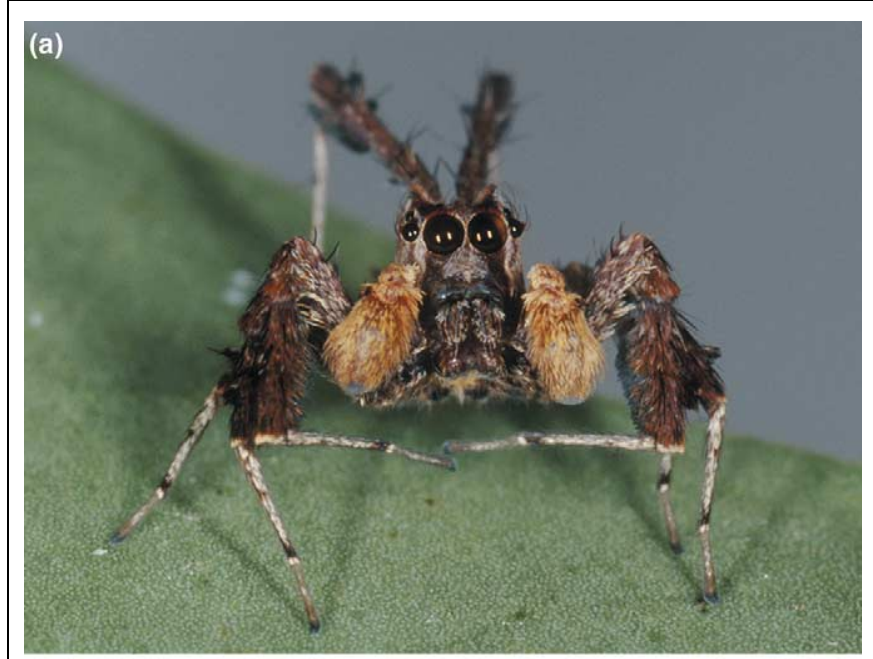

(b)

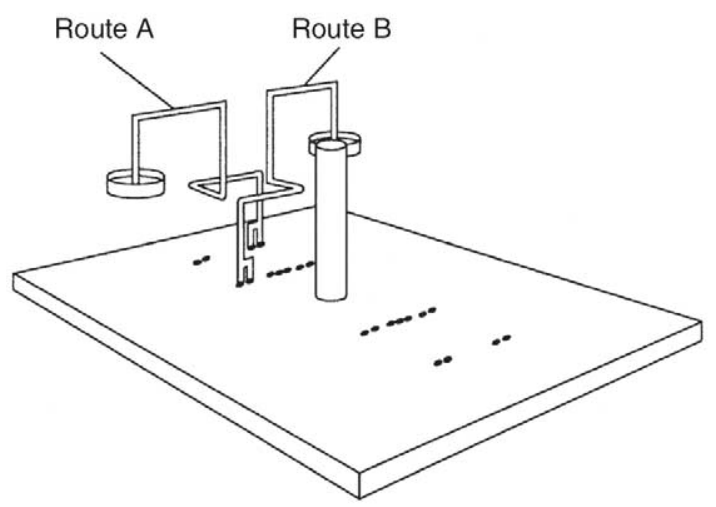

Figure 1. Jumping spider maze anticipation. (a) The jumping spider Portia fimbriata, close relative of $P$. labiata, showing their distinctive anterior median eyes. Photograph courtesy of R.R. Jackson. (b) One of many food maze configurations used to test the ability of jumping spiders to anticipate the correct route before actually walking it. A food lure is placed in one of the dishes at the end of either route $A$ or route $B$; the spider is placed on the starting platform and, after a suitable period for calming down, it begins to 'scan' the surroundings with its anterior median eyes before setting out towards the food. After leaving the starting platform and for the first portion of the route, the spider cannot see the lure or the dish. Reproduced, with permission, from Ref. [11]. insects without being strictly homologous $[13,14]$. Although not a case of place learning per se, the maze solving behavior of Portia spiders reveals a capacity for planning and anticipation that surpasses mere implicit memory.

Perception is the other face of cognition, and honeybees also excel in this faculty. They recognize illusory colors and contours when these are used as CS in associative conditioning paradigms that then test for recall with the authentic pattern stimuli $[15,16]$. Using a similar training strategy, honeybees have also shown the ability to distinguish abstract categories from displayed stimuli, such as symmetry versus asymmetry [17] and sameness versus difference [18] (Figure 2), and to generalize in the course of their training [19].

\section{The fruit fly}

The fruit fly would appear to be a cognitive poor cousin to the honeybee and jumping spider. But the same can be

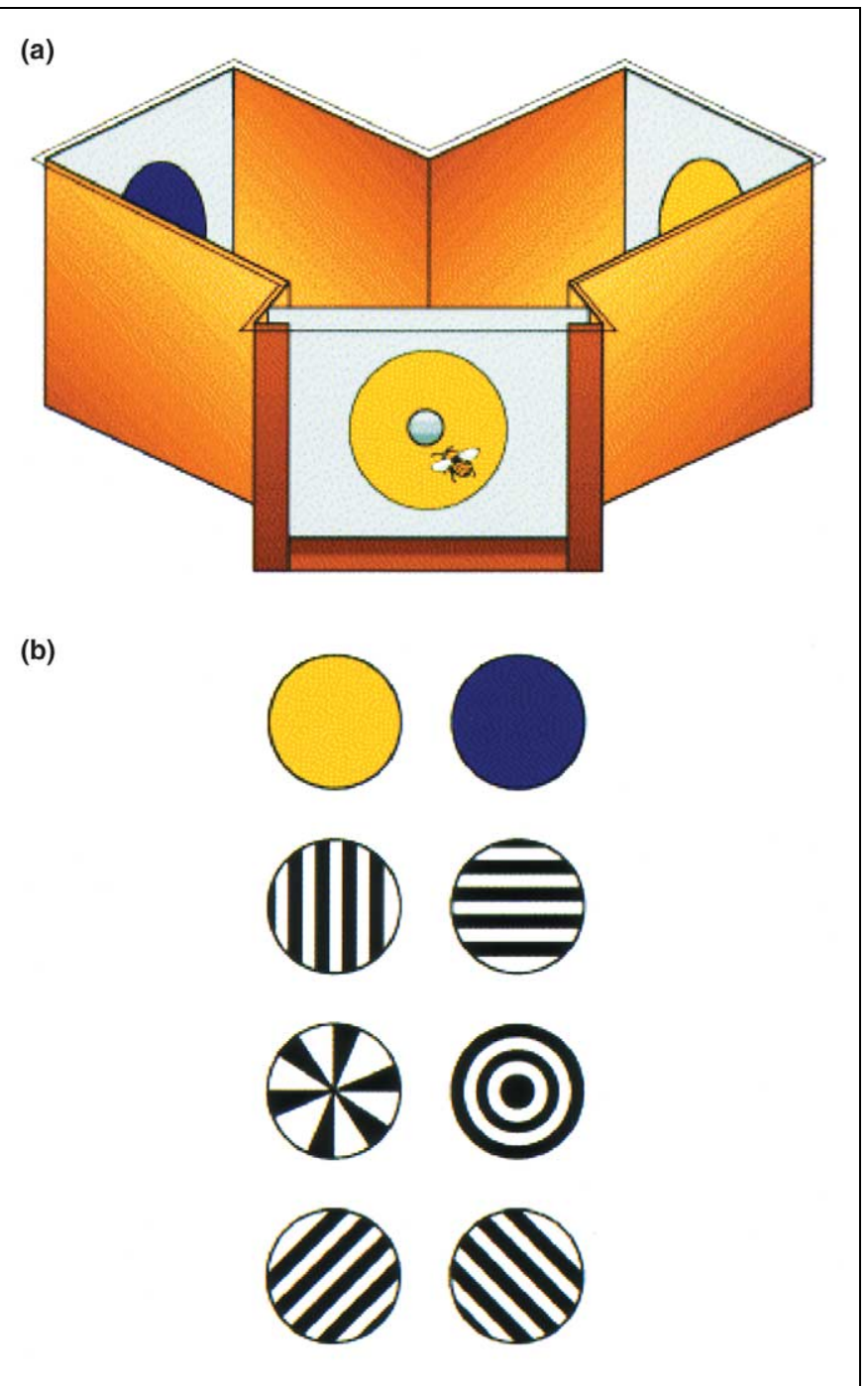

Figure 2. Honeybee pattern discrimination. (a) Choice maze for testing cognition in Apis mellifera. One pattern is displayed at the entrance to the maze. Inside, the same pattern is repeated in one arm and a different pattern displayed in the other arm. When trained to recognize 'difference' per se, honeybees will choose the pattern inside that differs from the one at the entrance. (b) Examples of patterns correctly distinguished by honeybees after being trained to recognize 'difference'. Reproduced, with permission, from Ref. [56]. 
said of virtually all other invertebrates, few, if any, of which have been studied in this way. Key to the apparent success of the honeybee and jumping spider is the ethological verisimilitude of the paradigms used in studying them. Because both species display cognitively sophisticated food foraging behavior, these behaviors became prime targets for further probing. Ethological observations of $D$. melanogaster do not suggest much in the way of sophisticated cognitive capability but, because of its powerful genetics, a handful of fly devotees have taken great pains to probe its cognitive potential [20-24]. The result is a growing repertoire that includes associative conditioning [21], incidental learning [22], contextual learning [23] and second-order conditioning [24]. Whether the fruit fly harbors additional, more sophisticated cognitive capabilities remains to be tested.

\section{How do they do it?}

Do these invertebrates accomplish such feats by an altogether different mechanism than we do? Or does their divergent anatomy subserve a functionally similar neural strategy of association, integration, abstraction and categorization? The answer to these questions can come only from direct analysis of real-time neural activity in the insect brain during cognitive and perceptual events.

Despite the high level of sophistication in evidence in their visual behaviors (and in the tests designed to reveal it), studies of the neural events taking place in the bee brain during visual recognition and perceptual events have been limited to physiological studies of retinal cells, of optic flow and of motion detection in the optic lobes. The occurrence of visual perception in the CNS has been inferred from behavioral tests. (Detailed and probing physiological studies of the circuitry subserving olfactory associative conditioning have been conducted in the honeybee $[9,25]$ in a paradigm that makes use of immobilized subjects, in contrast to the free flight required for high-level cognitive behavior in this insect. Similar studies on the circuitry of olfaction have also progressed significantly in other insects [26-28].)

\section{A physiological signature for 'attentiveness' in the fruit fly}

Ironically, it is in the smaller brain of the fruit fly that the most recent contribution to a systems physiology of visual perception in insects has appeared. (That these results were not obtained sooner is perhaps because the expectation for single-cell recording in fruit flies was never very great, although this has now been accomplished [29]). The recording of local field potentials (LFPs) under conditions where the fly exhibits a behavior analogous to selective attention reveals a signature for the 'attentive' response in the $20-30 \mathrm{~Hz}$ frequency range, and this physiological signature has been linked to the behavioral responses of flies to salient stimuli [30] (Figure 3). (Oscillations in this frequency range have previously been associated with olfactory responses in insects [28].) All of the sophisticated cognitive behaviors described previously in bees and spiders would appear to require attention-like mechanisms to restrict perception to the task at hand. It is likely that similar mechanisms govern behavior in the brains of (a)

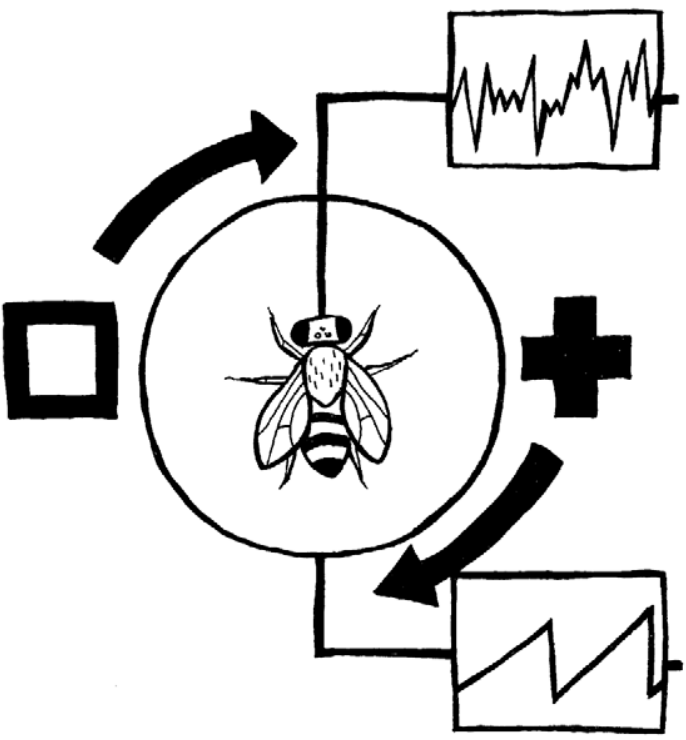

(b)

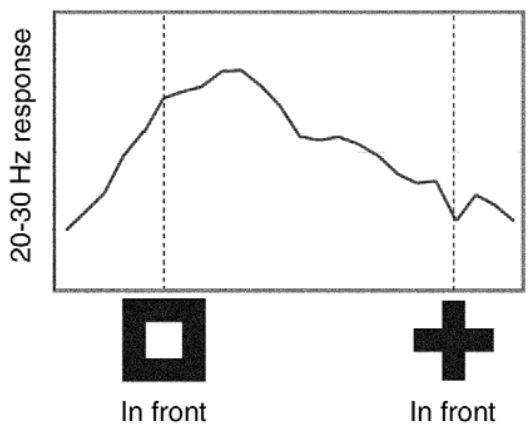

Position of image in arena

TRENDS in Neurosciences

Figure 3. Fruit fly selective discrimination. (a) Schematic drawing of the apparatus used to display two images to Drosophila melanogaster and to couple one with a salient heat stimulus. Local field potentials from the medial protocerebrum and the positions of the square and cross are recorded as the images rotate around the fly. (b) Characteristic recording of local field potential response in the $20-30 \mathrm{~Hz}$ range to the stimuli after pairing the square with heat. The response is selective for the square and maps to the front of the visual field of the fly. Drawings by B. van Swinderen.

bees, spiders and fruit flies alike. The physiological $20-30 \mathrm{~Hz}$ signature in fruit flies correlates well with the various features associated with attention-like behavior in mammals [31]: orientation, expectancy, stimulus differentiation and sustainability. The analytical value of a physiological 'percept' measure is its independence from behavioral output and from learning (which has been the principal report for perception). As just one example, it permits manipulations of the brain that can preclude behavioral output but nonetheless allow measurement of the percept. That this separation of physiological percept from behavior is biologically meaningful is suggested by the separation between perception and motor performance in anticipatory scanning of a maze by the jumping spider. In this phase of the behavior, they do not actually run the maze but instead focus selectively on particular aspects of it.

Orientation in the response of a fly is reflected behaviorally in its tracking of an image during tethered 
flight in a flight simulator [32], as well as in its physiological signature: the $20-30 \mathrm{~Hz}$ response to a rotating stripe is maximal when the fly initiates its tracking behavior [30]. Even when the stimulus is presented passively to the animal, the maximal $20-30 \mathrm{~Hz}$ LFP response maps to the front of the fly visual field, and the response timing and amplitude are a measure of stimulus salience. Expectancy is reflected in shifting of the maximal response to a rotating stripe to $0.5 \mathrm{~s}$ before arrival of the stripe in the front of the visual field of the fly, after pairing of an aversive stimulus with the stripe. Stimulus differentiation is displayed behaviorally and physiologically in the ability of flies to discriminate between two different images, either when differentially conditioned or when one is presented as a novel stimulus $[22,30]$. Sustainability is seen in the $\sim 20 \mathrm{~s}$ duration of the heightened LFP response after the stimulus is made more salient [30].

A neural correlate of expectancy is also seen in a subset of mushroom body neurons of the cockroach [33,34]. These neurons fire several hundred milliseconds before the onset of spontaneous locomotion and have been proposed to take part in the preparation for motor activity, by analogy to cells in mammalian cortex that anticipate movements [35]. The relationship of these cells to the $20-30 \mathrm{~Hz}$ LFPs recorded in the vicinity of the mushroom bodies of flies is not known.

The sustainability of the 'percept' signature in flies is a form of very short-term memory, on the scale of tens of seconds [30]. This highlights the importance of mechanisms of plasticity for actual perceptual events as well as for what we normally consider as short-term or long-term memory. In the honeybee, such short-term processes have been proposed to form a kind of working memory that is essential for learning contextual features [36]. Flies are clearly capable of short-term and long-term memory [37-39] but it is unclear how much use they actually make of it in their real world, where exploratory and novelty seeking strategies seem much more prominent than those involving sustained memory [40-43]. In this regard, it is also notable that the $20-30 \mathrm{~Hz}$ salience response of flies registers novelty as well as conditioning [30].

\section{Spatiotemporal correlations and perception}

Perceptual mechanisms of selective attention in mammals have been associated with temporal correlations in neuronal activity between different brain regions $[44,45]$. In monkeys, selective attention has been shown to correlate with specific patterns of coherent activity in cortical neurons [46], especially those firing in the gamma frequency $(35-80 \mathrm{~Hz})$ range $[47,48]$. Similarly, in a study of conscious perception in humans, using the alternating percepts produced in binocular rivalry, the conscious perception of an image is accompanied by a virtual explosion of coherent activity among distant sites all over the cortex [49].

The $20-30 \mathrm{~Hz}$ neural correlate of selective attention in the fruit fly brain shares this feature too. When a stimulus is being selected, following a conditioning paradigm designed to increase the salience of one of a pair of simultaneously presented stimuli, coherence in the $20-30 \mathrm{~Hz}$ frequency range between dorsal and medial brain regions increases significantly [30]. Moreover, the degree of coupling within the CNS serves as a measure of the state of vigilance of the fly, measured either directly as coherence between brain regions or indirectly as the correlation between protocerebral LFPs and movement. The degree of coupling (measured as coherence) within the brain increases when the fly is responding ('attending') to salient stimuli [30]. Further, the degree of coupling (measured as correlation) between brain LFP activity and bodily movement tracks with the degree of vigilance displayed by the fly: decreased behavioral responsiveness is associated with decreased coupling [50]. Vigilance thus appears to be a graded state depending on the degree of coupling between brain regions. The potential importance of coupling among brain regions extends to the insect brain a concept that has long been trenchant in mammalian systems neurobiology [44-49,51,52].

It is now clear that insects share a high degree of genetic homology with mammals [53], which matches the high degree of conservation of biochemical signalling mechanisms [54,55]. These findings originally came as a surprise, in part because of the almost complete lack of anatomical homology between insects and vertebrates. The phenomenological and mechanistic similarities in cognitive function between insects and mammals that are now coming to light suggest that, because brains in all organisms are made up of networks of neurons that communicate with each other through similar electrical and chemical signals, they might have only a limited number of strategies available to them for solving problems. If so, then the insect brain has even more to offer as a vehicle for understanding universal aspects of brain functioning (including our own) than previously thought.

\section{References}

1 von Frisch, K. (1923) Über die 'Sprache' der Bienen. Eine tierpsychologische Untersuchung: Zoologischer Jahrbücher (Physiologie) 40, 1-186

2 Squire, L.R. (1992) Memory and the hippocampus: a synthesis from findings with rats, monkeys, and humans. Psychol. Rev. 99, 195-231

3 Gould, J.L. (1986) The locale map of honey bees: Do insects have cognitive maps? Science 232, 861-863

4 Wehner, R. and Menzel, R. (1990) Do insects have cognitive maps? Annu. Rev. Neurosci. 13, 403-414

5 Menzel, R. et al. (2000) Two spatial memories for honeybee navigation. Proc. R. Soc. Lond. B. Biol. Sci. 267, 961-968

6 Menzel, R. et al. Honeybees navigate according to a map-like spatial memory. Proc. Natl. Acad. Sci. U. S. A. (in press)

7 Byl, N.N. et al. (1996) A primate genesis model of focal dystonia and repetitive strain injury: I. Learning-induced dedifferentiation of the representation of the hand in the primary somatosensory cortex in adult monkeys. Neurology 47, 508-520

8 Zhang, L.I. et al. (2002) Disruption of primary auditory cortex by synchronous auditory inputs during a critical period. Proc. Natl. Acad. Sci. U. S. A. 99, 2309-2314

9 Menzel, R. (2001) Searching for the memory trace in a mini-brain, the honeybee. Learn. Mem. 8, 53-62

10 Mizunami, M. et al. (1998) Mushroom bodies of the cockroach: their participation in place memory. J. Comp. Neurol. 402, 520-537

11 Tarsitano, M. and S. and Jackson, R.R. (1997) Araneophagic jumping spiders discriminate between detour routes that do and do not lead to prey. Anim. Behav. 53, 257-266

12 Tarsitano, M.S. and Andrew, R. (1999) Scanning and route selection in the jumping spider Portia labiata. Anim. Behav. 58, 255-265 
13 Strausfeld, N.J. and Barth, F.G. (1993) Two visual systems in one brain: neuropils serving the secondary eyes of the spider Cupiennius salei. J. Comp. Neurol. 328, 43-62

14 Strausfeld, N.J. et al. (1998) Evolution, discovery, and interpretations of arthropod mushroom bodies. Learn. Mem. 5, 11-37

15 Srinivasan, M. et al. (1987) Bees perceive illusory colours induced by movement. Vision Res. 27, 1285-1289

16 Horridge, G.A. et al. (1992) Insect perception of illusory contours. Philos. Trans. R. Soc. Lond. B Biol. Sci. 337, 59-64

17 Giurfa, M. et al. (1996) Symmetry perception in an insect. Nature 382, 458-461

18 Giurfa, M. et al. (2001) The concepts of 'sameness' and 'difference' in an insect. Nature 410, 930-933

19 Stach, S. et al. (2004) Local-feature assembling in visual pattern recognition and generalization in honeybees. Nature 429, 758-761

20 Quinn, W.G. et al. (1974) Conditioned behavior in Drosophila melanogaster. Proc. Natl. Acad. Sci. U. S. A. 71, 708-712

21 Tully, T. and Quinn, W.G. (1985) Classical conditioning and retention in normal and mutant Drosophila melanogaster. J. Comp. Physiol [A] 157, 263-277

22 Dill, M. and Heisenberg, M. (1995) Visual pattern memory without shape recognition. Philos. Trans. R. Soc. Lond. B Biol. Sci. 349, 143-152

23 Liu, L. et al. (1999) Context generalization in Drosophila visual learning requires the mushroom bodies. Nature 400, 753-756

24 Brembs, B. and Heisenberg, M. (2001) Conditioning with compound stimuli in Drosophila melanogaster in the flight simulator. J. Exp. Biol. 204, 2849-2859

25 Faber, T. et al. (1999) Associative learning modifies neural representations of odors in the insect brain. Nat. Neurosci. 2, 74-78

26 Galizia, C.G. et al. (2000) Calcium responses to pheromones and plant odours in the antennal lobe of the male and female moth Heliothis virescens. J. Comp. Physiol. [A] 186, 1049-1063

27 Laurent, G. (2002) Olfactory network dynamics and the coding of multidimensional signals. Nat. Rev. Neurosci. 3, 884-895

28 Daly, K.C. et al. (2004) Learning modulates the ensemble representations for odors in primary olfactory networks. Proc. Natl. Acad. Sci. U. S. A. 101, 10476-10481

29 Wilson, R.I. et al. (2004) Transformation of olfactory representations in the Drosophila antennal lobe. Science 303, 366-370

30 van Swinderen, B. and Greenspan, R.J. (2003) Salience modulates 20-30 Hz brain activity in Drosophila. Nat. Neurosci. 6, 579-586

31 Bushnell, P.J. (1998) Behavioral approaches to the assessment of attention in animals. Psychopharmacology (Berl) 138, 231-259

32 Wolf, R. and Heisenberg, M. (1980) On the fine structure of yaw torque in visual flight orientation of Drosophila melanogaster. II. A temporally and spatially variable weighting function for the visual field ('visual attention'). J. Comp. Physiol. 140, 69-80

33 Mizunami, M. et al. (1998) Mushroom bodies of the cockroach: activity and identities of neurons recorded in freely moving animals. J. Comp. Neurol. 402, 501-519

34 Okada, R. et al. (1999) Sensory responses and movement-related activities in extrinsic neurons of the cockroach mushroom bodies. J. Comp. Physiol. [A] 185, 115-129
35 Alexander, G.E. and Crutcher, M.D. (1990) Preparation for movement: neural representations of intended direction in three motor areas of the monkey. J. Neurophysiol. 64, 133-150

36 Menzel, R. (1999) Memory dynamics in the honeybee. J. Comp. Physiol. [A] 185, 323-340

37 Tully, T. et al. (1994) Genetic dissection of consolidated memory in Drosophila. Cell 79, 35-47

38 Siegel, R.W. and Hall, J.C. (1979) Conditioned responses in courtship behavior of normal and mutant. Drosophila. Proc. Natl. Acad. Sci. U. S. A. 76, 3430-3434

39 McBride, S.M.J. et al. (1999) Mushroom body ablation impairs shortterm memory and long-term memory of courtship conditioning in Drosophila melanogaster. Neuron 24, 967-977

40 Reif, M. et al. (2002) Evolutionary significance of courtship conditioning in Drosophila melanogaster. Anim. Behav. 63, 143-155

41 Tammero, L.F. and Dickinson, M.H. (2002) The influence of visual landscape on the free flight behavior of the fruit fly Drosophila melanogaster. J. Exp. Biol. 205, 327-343

42 Frye, M.A. et al. (2003) Odor localization requires visual feedback during free flight in Drosophila melanogaster. J. Exp. Biol. 206, 843-855

43 Tinette, S. et al. (2004) Cooperation between Drosophila flies in searching behavior. Genes Brain Behav. 3, 39-50

44 Sporns, O. et al. (1991) Modeling perceptual grouping and figureground segregation by means of active reentrant connections. Proc. Natl. Acad. Sci. U. S. A. 88, 129-133

45 Niebur, E. et al. (2002) Synchrony: a neuronal mechanism for attentional selection? Curr. Opin. Neurobiol. 12, 190-194

46 Steinmetz, P.N. et al. (2000) Attention modulates synchronized neuronal firing in primate somatosensory cortex. Nature 404, 187-190

47 Engel, A.K. and Singer, W. (2001) Temporal binding and the neural correlates of sensory awareness. Trends Cogn. Sci. 5, 16-25

48 Fell, J. et al. (2003) Is synchronized neuronal gamma activity relevant for selective attention? Brain Res. Brain Res. Rev. 42, 265-272

49 Srinivasan, R. et al. (1999) Increased synchronization of magnetic responses during conscious perception. J. Neurosci. 19, 5435-5448

50 van Swinderen, B. et al. (2004) Uncoupling of brain activity from movement defines arousal states in Drosophila. Curr. Biol. 14, 81-87

51 Merzenich, M. and M. and Kaas, J.H. (1980) Principles of organization of sensory-perceptual systems in mammals. Prog. Psychobiol. Physiol. Psychol. 9, 1-42

52 Seth, A.K. et al. Visual binding through reentrant connectivity and dynamic synchronization in a brain-based device. Cereb. Cortex (in press)

53 Rubin, G.M. et al. (2000) Comparative genomics of the eukaryotes. Science 287, 2204-2215

54 Hirth, F. and Reichert, H. (1999) Conserved genetic programs in insect and mammalian brain development. Bioessays 21, 677-684

55 Giudice, G. (2001) Conserved cellular and molecular mechanisms in development. Cell Biol. Int. 25, 1081-1090

56 Giurfa, M. (2003) Cognitive neuroethology: dissecting non-elemental learning in a honeybee brain. Curr. Op. Neurobiol. 13, 726-735

Forthcoming Conference

\author{
Alzheimer's and Parkinson's Diseases: Insights, Progress and Perspectives \\ 7th International Conference AD/PD 2005 \\ Sorrento, Italy \\ March 9-13, 2005
}

Secretariat: Kenes International

Tel: +41229080488

Fax: +4122732 2850

email: adpd@kenes.com

website: www.kenes.com/adpd 